\title{
Las Américas, ¿continente en crisis?
}

Americas, a continent in crisis?

Les Amériques, continent de la crise?

Florence Pinot de Villechenon, Jacques Pothier y Carlos Quenan Traductor: Marta Gómez

\section{(2) OpenEdition}

1 Journals

\section{Edición electrónica}

URL: https://journals.openedition.org/ideas/3065

DOI: 10.4000/ideas.3065

ISSN: 1950-5701

Este artículo es una traducción de:

Les Amériques, continent de la crise ? - URL : https://journals.openedition.org/ideas/605 [fr]

Otras traducciones del artículo:

Americas, a continent in crisis? - URL : https://journals.openedition.org/ideas/3060 [en]

As Américas, um continente em crise? - URL : https://journals.openedition.org/ideas/4697 [pt]

Editor

Institut des Amériques

Referencia electrónica

Florence Pinot de Villechenon, Jacques Pothier y Carlos Quenan, «Las Américas, ¿continente en crisis?», IdeAs [En línea], 4 | 2013, Publicado el 21 junio 2013, consultado el 20 octubre 2022. URL: http://journals.openedition.org/ideas/3065 ; DOl: https://doi.org/10.4000/ideas.3065

Este documento fue generado automáticamente el 20 octubre 2022.

\section{(c) $)(9)$}

Creative Commons - Atribución-NoComercial-SinDerivadas 4.0 Internacional - CC BY-NC-ND 4.0 https://creativecommons.org/licenses/by-nc-nd/4.0/ 


\title{
Las Américas, ¿continente en crisis?
}

\author{
Americas, a continent in crisis? \\ Les Amériques, continent de la crise? \\ Florence Pinot de Villechenon, Jacques Pothier y Carlos Quenan \\ Tradución : Marta Gómez
}

1 El primer decenio de este tercer mileno se cerrará con un Occidente en crisis. ¿Crisis del sistema capitalista? ¿Crisis del desarrollo en su versión occidental? ¿Crisis de los valores que representa Occidente? En su expansión, esta crisis global ha golpeado sobre todo a Europa y sigue sacudiendo a la zona euro, e incluso al modelo europeo, en sus cimientos. Sin embargo, fue de América de donde partió la onda expansiva provocada por la quiebra de Lehman Brothers en septiembre de 2008.

2 En efecto, a partir de 2007 y de la crisis de confianza en los créditos inmobiliarios americanos, la crisis que se prolonga hasta 2013 tiene su origen en América, al igual que la de 1929, la crisis emblemática. ¿Será América el continente de «la crisis»? La pregunta es tentadora. Desde el siglo XIX hasta la primera mitad del siglo XX, el capitalismo ha vivido sus crisis más violentas en Estados Unidos. En cuanto a América Latina, aún recordamos, en las últimas décadas del siglo XX, las graves turbulencias que atravesaron los países de la región, enfrentados al azote de la hiperinflación y el endeudamiento, sin olvidar las convulsiones sociales y la inestabilidad políticoinstitucional.

3 La presente edición es fruto de una reflexión cuyo punto de partida es el coloquio anual del Instituto de las Américas (IdA) organizado en la Sorbona los días 19 y 20 de noviembre de 2009 por el IHEAL-Universidad París 3, la Universidad de Versailles SaintQuentin-en-Yvelines y el CERALE-ESCP Europe (Centro de Estudios e Investigación América Latina-Europa) sobre el tema «Las Américas frente a la crisis». Una vez lanzada la problemática, diversos eventos académicos como los seminarios del IdA «Economías y economistas de las Américas» y la conferencia organizada por el CERALE el 16 de noviembre de 2011 sobre «Inflación y gestión monetaria de las crisis en el seno del G20», entre otros, han contribuido a enriquecer la reflexión. 
4 Analizar la crisis desde la perspectiva americana no está exento de sentido, sobre todo, en el marco de IdeAs, publicación insignia del Instituto de las Américas. El continente que se extiende desde Alaska hasta Tierra de Fuego muestra el rostro de un Occidente complejo y «extremo» en el que durante más de cien años el capitalismo de la potencia central y hegemónica del siglo XX ha convivido con el capitalismo "periférico» latinoamericano para, posteriormente, ver aparecer en este inicio de milenio a los nuevos países emergentes con Brasil a la cabeza.

El objetivo de los textos reunidos en este número no es tanto zanjar la cuestión planteada anteriormente buscando constantes continentales detrás de los cambios que se están fraguando como ofrecer, de Norte a Sur, miradas diversas sobre las rupturas en curso y medir su impacto. ¿Podría ser la crisis la prueba reveladora que permitiría poner de manifiesto una identidad americana? $¿$ O hay que verla como una etapa de una cronología americana totalmente abocada a lo apocalíptico? Más que buscar una coherencia continental allí donde ha habido y sigue habiendo situaciones y niveles de vida y de desarrollo dispares, los autores analizan la crisis en las Américas en sus diversas dimensiones (económica, social, política, etc.). En cuanto al horizonte temporal, si bien el año 2009 constituye una fecha crítica, algunos autores se remontan tiempo atrás para ilustrar sus tesis mientras otros adoptan un enfoque deliberadamente prospectivo que necesariamente va más allá del tiempo presente.

Es evidente que el capitalismo está en crisis. Lo que hasta ahora ha servido de modelo asociado a Occidente tanto desde el punto de vista económico y político como social, e incluso como civilización, ha perdido parte de su poder de atracción para el resto del planeta. Desindustrialización, desempleo, desigualdades, partidos políticos desacreditados, ambiente de malestar... no son motivo de ilusión para nadie pese a que el apetito de consumo de los países emergentes induce a pensar que el impulso para alcanzar «el modelo» no ha perdido fuerza.

7 La noción de «crisis», dada su omnipresencia, plantea un problema de conceptualización al que Laurence Whitehead trata de dar respuesta. En su artículo «Crisis in the Americas: Is there a Regionally Distinctive Kind?», la autora presenta algunas pistas sobre la forma de aprehender el concepto y sobre la especificidad de las Américas, si la hay, frente a las crisis. Los textos siguientes buscan, en primer lugar, una lectura global de la crisis. Jean-Luc Tendil, por ejemplo, analiza las crisis norteamericanas a la luz de los últimos ciento setenta años ( $«$ Crisis en Estados Unidos desde 1837: factores para nuevos brotes y expansión») mientras Carlos Quenan («América Latina frente a la crisis económica internacional: buena resistencia global y diversidad de situaciones nacionales») propone una lectura latinoamericana de la crisis. Este texto persigue más de un objetivo al centrarse no solo en los efectos de la crisis sino también en su carácter pedagógico, ilustrado a través del análisis de la reacción de los Estados: ¿podemos sacar enseñanzas para el futuro de la gestión macroeconómica de la crisis en América Latina? ¿Podemos prepararnos mejor para hacer frente a crisis venideras? En esta línea se inscribe también el artículo «Paying your Visa with your Master Card: la espiral infernal de las tarjetas de crédito en Estados Unidos», de Marie-Christine Pauwels, que analiza el impacto de la crisis en un sector específico de la actividad económica, política y académica desde el punto de vista del endeudamiento de los particulares. Por su parte, Jean-Baptiste Velut, en su artículo «Estados Unidos y la "otra crisis": la ayuda exterior estadounidense puesta a prueba por la crisis alimentaria mundial», se centra por un lado en el papel de los agentes políticos en Estados Unidos frente a la crisis alimentaria 
de 2008 y, por otro, en los mecanismos de la ayuda exterior estadounidense. Carole Massey-Bertonèche no se contenta con ilustrar los efectos de la crisis en un ámbito concreto sino que analiza además la respuesta de la estructura estatal a los problemas planteados por la crisis en la educación superior.

8 Seguidamente se da paso a la narración de historias, sobre todo de ficción, sobre la crisis. Es el caso del artículo de Jacques Pothier titulado «De la crisis intrínseca a la crisis radical en Estados Unidos: una lectura cultural», que resitúa la crisis actual en la historia de las representaciones literarias de la crisis en Norteamérica, desde el apocalipsis puritano. André Cartapanis, en su análisis prospectivo sobre los «Retos monetarios y financieros de la post-crisis en América Latina» conduce al lector al periodo posterior a la crisis. El autor se interroga sobre el impacto que en el futuro tendrán los mecanismos anticíclicos creados en América Latina, menos golpeada que otras regiones del mundo pero donde la vigilancia sigue vigente habida cuenta de la fragilidad existente. Por último, Florence Pinot de Villechenon («Los perímetros americanos frente a la crisis y a la globalización») se centra en el papel que ha tenido la crisis en la recomposición de los espacios regionales y subregionales americanos y en el establecimiento de nuevos equilibrios geopolíticos.

9 Esta reflexión sobre la integración en las Américas se refleja en el texto de Bernardo Sorj, «A dinâmica Regional além dos mitos», en la sección «Enfoques». En esta misma sección, el artículo de Jean-Michel Saussois titulado «Las soluciones para salir de la crisis están en crisis» aborda la realidad de un sistema capitalista en crisis y explora las vías de salida. Le toca a Edgar Morin («Las Américas frente a la crisis: exposición») cerrar este número con una reflexión sobre las creencias desbaratadas, los cuestionamientos, las contradicciones y las rupturas dentro de las sociedades americanas.

10 El presente número, por tanto, aborda las crisis y sus efectos en el continente americano a través de un enfoque deliberadamente diverso: ¿Cómo surgen las crisis? ¿Cuáles son sus efectos? ¿Qué estrategias de respuesta suscitan? ¿Qué enseñanzas sacar? El objetivo de los autores no es otro que poner a disposición del lector elementos que permitan una mejor comprensión de los fenómenos de crisis en el continente e ilustrar cómo contribuyen las crisis americanas a la comprensión de la crisis mundial. De ahí por tanto este elenco de contenidos del que derivan lenguajes plurales, así como expresiones, manifestaciones y representaciones diversas de la crisis.

11 Se invita al lector a una lectura ecléctica, con sus desfases temporales y espaciales. Lejos de los autores la pretensión de dibujar un panorama sombrío tratando de configurar el futuro, sino más bien el deseo de permitir al lector comprender mejor los cambios en curso.

12 Para dar continuidad a este número, IdeAs abordará en su próxima edición de diciembre de 2013 las manifestaciones sociales de la crisis y el impacto de ésta en el mundo laboral: temas como los cambios en el trabajo asalariado, la evolución de la actividad sindical en Estados Unidos, las estrategias de respuesta de una categoría profesional (los menores) en Bolivia, entre otros, serán analizados en profundidad y aportarán luz sobre la forma de ver y organizar el trabajo en las Américas. 


\section{AUTORES}

\section{FLORENCE PINOT DE VILLECHENON}

Florence Pinot de Villechenon es profesora asociada en ESCP-Europe, donde coordina la cooperación académica con América Latina y dirige el CERALE (Centro de Estudios e Investigación América Latina-Europa). Egresada de la Universidad de Buenos Aires y de la Universidad Paris IVSorbonne, obtuvo su doctorado en la Universidad Paris VII. Sus trabajos de investigación se centran en la política latinoamericana de la UE y de los países miembros, las relaciones EuropaAmérica Latina, las estrategias de internacionalización de las empresas europeas particularmente las PYMES- en América Latina y la gestión de grandes proyectos culturales internacionales. Florence Pinot de Villechenon también imparte clases en el IHEAL y trabaja como experta en cooperación académica para los ministerios franceses de Asuntos Exteriores y de Educación. Es miembro de la Chambre du Brésil en France. Entre sus publicaciones figuran: « Cooperación descentralizada Europa-América Latina: contribuciones de la política de cooperación comunitaria a la consolidación de la democracia en Latinoamérica », Política pública y democracia en América Latina, F. Maríñez y V. Garza Cantú ed., Ed. Porrúa, 2009 ; L'Argentine, terre d'investissement ?, (Ed.) Harmattan, Paris, 2008 ; « La latinidad: una cuestión de afinidad cultural en las relaciones eurolatinoamericanas », Homenaje a Carlos A. Ronchi March, Universidad de Buenos Aires, 2003 ; Fêtes géantes. Les expositions universelles pour quoi faire?, (Ed.) Autrement, coll. Essais, 2000. Pinot@escpeurope.eu

\section{JACQUES POTHIER}

Jacques Pothier enseigne la littérature nord-américaine à l'Université de Versailles Saint-Quentin en Yvelines, où il dirige le laboratoire de recherche "Suds d'Amériques". Il est directeur de l'Institut des Langues et des Études Internationales. Il est l'un des vice-présidents de l'Institut des Amériques. Il est l'auteur de deux monographies, William Faulkner : essayer de tout dire, Paris, Belin, 2003 ; et Les nouvelles de Flannery O'Connor, Nantes, France, Le Temps, 2004). Ses champs de recherche sont la littérature du Sud des États-Unis, l'aire culturelle méso-américaine, le modernisme et le post-modernisme dans les arts narratifs et visuels, l'épistémologie des études américaines, le rôle de la littérature dans la construction d'identités ethniques, locales ou nationales et terrain privilégié de transferts culturels. Il participe à l'édition des œuvres de Faulkner dans la Bibliothèque de la Pléiade, chez Gallimard. jacques.pothier@uvsq.fr

\section{CARLOS QUENAN}

Carlos Quenan est professeur des universités en sciences économiques à l'Institut des Hautes Etudes de l'Amérique latine (IHEAL, Université de Paris III Sorbonne Nouvelle). Il est également chercheur au CREDA - Unité mixte de recherche Université Sorbonne Nouvelle/CNRS-, où il est responsable de l'axe «Intégration régionale ».

Vice-président Amérique latine à l'Institut des Amériques, il est également co-président de la Section Europe/Amérique latine de la Latin American Studies Association (LASA).

Il est auteur ou co-auteur d'une soixantaine de livres et de nombreux articles scientifiques et de divulgation , notamment dans le domaine des relations économiques internationales, des relations Europe/Amérique latine, de l'intégration régionale et de la macroéconomie et des politiques économiques des pays émergents, principalement latino-américains. En outre, il a été ou est professeur invité dans de nombreuses institutions universitaires et de recherche d'Amérique latine, États-Unis, Canada, Europe et Afrique, et est membre du comité 
éditorial de nombreuses revues et publications spécialisées et de diverses instances internationales de recherche.

Il a été ou est consultant auprès de nombreuses institutions publiques internationales, nationales des pays en développement, ainsi qu'auprès d'instances publiques et privées en Europe et en France (notamment consultant permanent au service de la recherche économique à Natixis). quenan@wanadoo.fr 